\title{
NUREKSAIN WANGUN INTRINSIK MIWAH AJI PANGAJAH- AJAHAN TATA LAKSANA RING PUPULAN SATUA BAWAK AWENGI RING HOTEL SENTRAL KAKAWIAN I GUSTI PUTU SAMAR GANTANG
}

\author{
Ni Kt. Eria Puspayanti ${ }^{1}$, I.B.M. Ludy Paryatna ${ }^{1}$, I.A. Sukma Wiriani ${ }^{2}$ \\ Program Studi Pendidikan Bahasa Bali \\ Universitas Pendidikan Ganesha \\ Singaraja, Indonesia
}

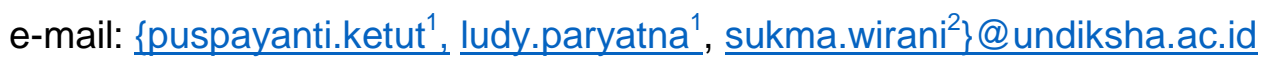

\begin{abstract}
Kuub
Tetilikan puniki matetujon anggen nlatarang (1) wangun intrinsik miwah (2) aji pangajahajahan tata laksana ring buku pupulan satua bawak "Awengi ring Hotel Sentral" kakawian I Gusti Putu Bawa Samar Gantang. Jejering ring tetilikan puniki inggih punika buku pupulan satua bawak "Awengi ring Hotel Sentral" kakawian I Gusti Putu Bawa Samar Gantang lan penandang ring tetilikan puniki inggih punika wangun intrinsik lan aji pangajah-ajahan tata laksana. Tetelikan puniki nganggen tetilik deskritif kualitatif lan mupulang datanyane kalaksanayang nganggen kramaning dokumentasi. Data tureksa sane kaanggen inggih punika deskritif kualitatif, pah-pahannyane (1) reduksi data, (2) ngwaderang data, (3) makarya panyutet. Pikolih ring tetilikan puniki, kapertama wangun intrinsik sane wenten ring buku pupulan satua bawak Awengi ring Hotel Sentral inggih punika, unteng, lelintihan, pragina miwah pawatekan, desa kala patra, genah sang pangawi, piteket, miwah paribasa. Kaping kalih aji pangajah-ajahan tata laksana sane wenten ring buku pupulan satua bawak "Awengi Ring Hotel Sentral" kakawian I Gusti Bawa Samar Gantang inggih punika sradha bhakti, seneng ngawacen, masawitra, arjawa, meled uning, swadarman, jemet makarya, awiwahara, ngajiang kawagedan, miwah tresna asih. Cutetnyane tetilikan ring buku pupulan satua bawak "Awengi ring Hotel Sentral" puniki sampun kewangun olih wangun intrisik sane jangkep, tur aji pangajah-ajahan tata laksana sane mapaiketan sareng kawentenan lan parilaksana masimakrama.
\end{abstract}

Kruna Jejaton: intrinsik, aji pangajah-ajahan tata laksana

\section{abstrack}

This study aims to describe (1) intrinsic elements and (2) the value of character education in the book collection of short stories "Awengi ring Hotel Sentral" by I Gusti Putu Bawa Samar Gantang. The subject of this study is the book collection of short stories "Awengi ring Hotel Sentral" by I Gusti Putu Bawa Samar Gantang and the object of this research is the intrinsic element and the value of character education. This study uses descriptive qualitative techniques and the data collection method used is documentation. Data analysis methods used are (1) data reduction, (2) presenting data, (3) conclusions. The results of the first study regarding the intrinsic elements in the book collection of short stories "Awengi ring Hotel Sentral" are themes, plot, character and characterization, setting or setting, point of view, mandate, language style. The second is the value of character education in the book "Awengi ring Hotel Sentral" short story by I Gusti Putu Bawa Samar Gantang to religious, love to read, friendly or communicative, honest, curiosity, responsibility, hard work, tolerance, respect for achievement, and love peace. The research conclusions from this book collection of "Awengi ring Hotel Sentral" short stories are 
formed by complete intrinsic elements and character education values that have to do with the existence of community behavior.

Keywords: Intrinsic, character education

\section{PURWAKA}

Parajana Baline akeh pisan ngelimbakang lan nelebin kria sastra. Wenten sane nelebin sastra kanggen ngrereh pangupa jiwa sakadi makarya satua bawak, makarya puisi miwah sane tiosan sane kasurat ring orti Bali. Sastra sane katelebin kaanggen nincapang parisolah sane becik, santukan akeh madaging aji pangajah-ajahan tata laksana. Kanggen nglimurang manah utawi ngicalang rasa sebet lan lesu ritatkala ngemargiang swagina sane lianan minakadi matembang sakadi sekar rare, sekar alit, sekar madya, lan sekar agung. Makudang-kudang daging cakepan sastra sane wenten ring Bali kasengguh kasusastraan Bali.

Manut aabnyane kasusastraan Bali punika kakepah dados kalih soroh luir punika kasusastraan Bali purwa lan kasusastraan Bali anyar.Kawentenan kesusastraan Bali purwa kantun maurip ring wewidangan parajanane, santukan kantun kaanggen ring sakancan upakara yadnya. Lian ring kasusastraan Bali purwa, kawentenan kesusastraan Bali anyar kantun arang kaanggen ring kauripan parajanane. Yening selehin kria sastra Bali anyar saking daging satuanyane pateh sekadi daging sastra Bali purwa, akeh madaging guna sarat sane prasida kaanggen dasar ring kauripan. Kesusastraan Bali anyar kakepah dados kalih, inggih punika wenten gancaran (satua bawak, novel, drama) lan sane kaping kalih punika wenten puisi Bali anyar. Silih sinunggil imba sastra Bali anyar inggih punika satua bawak. Satua bawak (cerpen) pinaka sarana sane prasida kaanggen ngicen pangajah-ajah majeng ring para alit-alit miwah para yowanane anggen dasar maparilaksana ring kauripan.

Manut (Sumardjo lan Saini, 1997:37) maosang satua bawak inggih punika satua sane mawit awi-awian (fiktif) miwah ketah satuanyane bawak. Pupulan satua bawak "Awengi ring Hotel Sentral" puniki sinunggil satua bawak sane kakaryanin olih I Gusti Putu Bawa Samar Gantang sane satuanyane nyihnayang ring sajeroning kahuripan manusane. Pupulan satua bawak puniki akehnyane 12 satua bawak. Pupulan satua bawak "Awengi ring Hotel Sentral" puniki kaambil olih panilik sane kaanggen tetilikan, santukan dagingdaging satuanyane nyritayang indik kahuripan manusa. Sane nyihnayang punika prasida kacingak ring satua bawak sane mamurda 'Oh Diani'. Dagingnyane nyritayang indik anak lanang sane mawastan Gde Mangku sane buduh kasmaran kapining anak istri mawasta Diani, rasa senengne kapining anak istrine sane keliwat, Gde ngantos lali tekening paplajahan. Ngantos dewekne nemu sengkala tabrak motor santukan gedegnyane sane kaliwat nepuk anak luh punika magandengan ngajak anak muani lianan.

Manut sane sampun katlatarang ring ajeng, panilik meled ngambil pupulan satua bawak "Awengi ring Hotel Sentral" kekawian I Gusti Putu Bawa Samar Gantang sane kaanggen tetilikan. Akeh satua bawak puniki wenten 12 inggih punika 'Leak ring Bukit Pecatu', 'Awengi ring Hotel Sentral', 'Sang Bayu Nyarengin Antaka', 'Bukit Rangda', 'Pak Putu Joni', 'Kursi Roda', 'Oh Diani', 'Teroris Majenggot Kambing', 'Sandikala ring Pasisi Kelanting', 'Kangen Titiang Maring Bali', 'Sinengkawonan', 'Puisin Tiang Puisin Ragane' nanging sane pacang ketilikin penilik wantah 5 sane mamurda 'Leak ring Bukit Pecatu', 'Pak putu Joni', 'Kursi Roda', 'Oh Diani', miwah 'Sinengkawonan' puniki sane pacang katilikin.

Penilik ngambil pupulan satua ring ajeng anggen tetilikan, santukan pupulan satua ring ajeng puniki madue 
wangun intrinsik taler madaging aji pangajah-ajahan tata laksana sane pacang keanggen sasuluh majeng ring para sisia lan para yowanane maparilaksana sane becik. Tiosan ring punika lima murda satua sane katilik panilik puniki madue satua sane banget nudut manah pangwacen napi malih satua-satuanyane dangan karesepang olih pangwacen miwah pinilik. Sakemaon panilik nenten ngambil murda satua Awengi ring Hotel Sentral santukan daging satuanyane nenten patut katilikin, manut panilik ring daging satuanyane nenten kapolihang aji pangajah-ajahan tata laksana sane patut anggen para sisia. Daging satua puniki nenten patut kawacen olih para sisia, santukan wenten kruna-kruna sane nenten patut kawacen olih para sisia, tiosan ring punika konflik utawi pikobet ring satua Awengi ring Hotel Sentral nenten kadagingin. Punika ngawinan panilik gentosing antuk murda satua sane lianan, sane wenten ring buku pupulan satua bawak Awengi ring Hotel Sentral puniki. Panilik gentosin antuk murda satua sane madue daging satua sane dangan karesepang, daging satuanyane madue kruna-kruna sane patut kawacen olih para sisia lan madue aji pangajah-ajahan tata laksana sane patut katulad.

Pupulan satua bawak awengi ring hotel sentral puniki becik kewacen santukan akeh aji pangajah-ajahan sane prasida katulad. Kria sastra satua bawak puniki taler kawangun antuk wangun intrinsik lan ekstrinsik pateh sakadi wangun satua sane siosan. Wangun intrinsik punika wangun ring daging satua sakadi: 1) Unteng, 2) Lelintihan, 3) Watak, 4) Pragina, 5) Latar (desa kala patra), 6) Paribasa, miwah 7) Tetuek utawi Pabesen. Yening wangun ekstrinsik punika sane ngiusin daging satua saking palemahan sang pangawi, sakadi: 1) Biografi indik pangawinyane, lan 2) indik guna sarat utawi aji pangajah-ajahan.

Pupulan satua bawak sane
mamurda "Awengi ring Hotel Sentral"punika sampun nudut manah sang panilik, napi malih daging satuanyane. Nika mawinan panilik meled
Saking dadalan pikobet punika panilik ngalaksanayang tetilikan sane mamurda "Nureksain Wangun Intrinsik miwah Aji Pangajah-Ajahan Tata Laksana ring Pupulan Satua Bawak Awengi ring Hotel Sentral Kekawian I Gusti Putu Bawa Samar Gantang".

Manut dadalan pikobet ring ajeng, bantang pikobet ring tetilikan puniki inggih punika (1) Sapunapi wangun intrinsik pupulan satua bawak sane mamurda Awengi ring Hotel Sentral? (2) Aji pangajah-ajahan tata laksana napi manten sane wenten ring pupulan satua bawak sane mamurda Awengi ring Hotel Sentral?

Tetujon tetilik puniki inggih punika (1) Prasida nelataran wangun intrinsik saking pupulan satua bawak Awengi ring Hotel Sentral kekawian I Gusti Putu Bawa Samar Gantang. (2) Prasida nelatarang aji pangajah-ajahan tata laksana sane wenten saking pupulan satua bawak Awengi ring Hotel Sentral kekawian I Gusti Putu Bawa Samar Gantang. Kawigunan ring tetilik puniki ka kepah dados kalih inggih punika kawigunan teoritis lan kawigunan praktis. Kawigunan teoritis inggih punika mangda daging sane wenten saking pupulan satua bawak awengi ring hotel sentral punika prasida karesepin malarapan antuk wangun intrinsik lan aji pangajah-ajahanan tata laksana sane kawentenang. Kawigunan praktis katujuang majeng krama Bali, pangawacen, lan panilik tiosan.

Sepat siku-siku sane kaanggen ring sajeroning ngamargiang tetilikan puniki inggih punika (1) Kesusastran Bali, (2) Teges Satua Bawak, (3) Cecirin Satua Bawak, (5) Soroh-soroh Satua bawak, (4) Wangun Sastra, (5) Aji Pangajah-Ajahan Tata Laksana.

Kasusastraan inggih punika kaweruhan sane becik tur metu saking manah utawi pikayunan para pangawi sane nganggen aksara Bali miwah aksara latin pinaka piranti sasuratannyane (Antara, 2009: 1). Tiosang ring punika, Gautama (2007: 2) maosang kesusastraan Bali inggih punika palaning reriptaan Ida Sang Kawiswara malarapan antuk kayun lan pawisik (ilham utawi wahyu) sane kaiket 
antuk basa utawi aksara sane lengut. Prasida kacutetang kasusastraan inggih punika mateges kawentenan ajah-ajah utawi kaweruhan sane becik tur mabuat. Kametuang saking budi jnana utawi saking manah para pengawi ring Bali sane madados piranti sasuratanyane aksara Bali miwah aksara latin sane nganggen basa Bali.

Manut Antara (2011:11) satua bawak punika soroh sastra Bali marupa gancaran sane susunan panyuratannyane nganutin srana cerita pendek Indonesia, tur ngundukang tetuek reraosannyane wantah akidik. Taler Sumardjo (2001: 91) nampeninin parindikan satua bawak punika pinaka seni, kawagedan nguningayang satua, jangkep masikian, lan sami pahpahannyane kasaratang, nanging wenten taler pah-pahan sane akeh. Manut Nurgiyantoro (1998: 10) satua bawak inggih punika sinunggil satua sane prasida kawacen ategakan utawi awai manten nenten sue nelasang galah, kiranglangkung prasida nelasang galah wantah tengah jam ngantos duang jam nenten sakadi ngawacen novel sane nelasang galah sue tur nenten telas kawacen awai utawi ategakan.

Malarapan antuk napi sane sampun katlatarang ring ajeng prasida kacutetang satua bawak inggih punika wangun kria sastra gancaran sane marupa satua saking sikian parindikan ring kahuripan manusa, tur wantah nelasang galah tengah jam ngantos duang jam anggen ngawacen satua ring satua bawak.

Ring satua bawak madue kalih wangun sastra pateh sakadi kria sastrakria sastra sane lianan inggih punika wangun intrinsik lan wangun ekstrinsik. Suroto (1989: 88) nelatarang wangun intrinsik inggih punika unsur-unsur sane ngwangun kria sastra minakadi (1) unteng (tema), (2) lelintihan satua (alur), (3) pragina miwah pawatekan, (4) desa, kala miwah patra (latar utawi setting), (5) Genah sang pangawi (sudut pandang) (6) piteket (amanat), (7) paribasa (gaya bahasa). Wangun ekstrinsik punika pianaka subjektivitas individu pengawi sane madue tikas, keyakinan lan pandangan hidup ring makasamian punika pacang ngweruhin kria sastra ri tatkala kasurat. Wangun ekstrinsik taler wenten biografi pengarang lan psikologi sane pateh ngwangun kria sastra punika (Wellek lan Warren ring Burhan, 1998:24).

Yaumi (2014: 83) maosang wenten 18 indik aji pangajah-ajahan tata laksana inggih punika sradha bhakti (religius), arjawa (jujur), awiwahara (toleransi), manut ring tata lakasana (disiplin), jemet makarya (kerja keras), akeh madue reragran (kreatif), sida ngraga (mandiri), briak briuk (demokrasi), meled uning (rasa ingin tahu), prawira negara (semangat kebangsaan), tresna ring pratiwi (cinta tanah air), ngajiang kawagedan (menghargai prestasi), masawitran (bersahabat/komunikatif), tresna asih (cinta damai), seneng ngwacen (gemar membaca), urati ring palemahan (peduli lingkungan), urati krama (peduli sosial), lan nganutin swadharma (tanggung jawab).

\section{KRAMANING TETILIK}

Tetilikan puniki ngranjing ring tetilikan deskriptif kualitatif. Kramaning tetilik puniki wenten lelima sane pacang kawedarang sakadi: (1) palihan tetilik, palihan tetilik cutetnyane wantah ngarencanayang indik parilaksana sadurung kalaksanayang, minakadi mupulang, nyajiang, lan nyelehin data ring tetilikan. (2) jejering lan panandang tetilik, jejering ring tetilikan puniki inggih punika pupulan satua bawak Awengi Ring Hotel Sentral. Penandang tetilikan puniki inggih punika wangun intrinsik lan aji pangajah-ajahan tata lakasana. (3) piranti tetilik, piranti tetilik inggih punika sakancan piranti sane kaaggen mupulang data ring sajeroning tetilik. Piranti tetilik sane kaanggen ring tetilikan puniki inggih punika kartu data. Kartu data puniki madaging kode data, murda satua, sumber data, wangun intrinsik miwah aji pangajah-ajahan tata lakasana sane wenten ring pupulan satua bawak Awengi Ring Hotel Sentral. (4) mupulang data, mupulang data sane 
kaanggen ring tetilikan puniki inggih punika kramaning dokumentasi santukan tetilikan puniki marupa teks bacaan utawi buku. (5) data tureksa, pamargi sane pacang kalaksanayang ring sajeroning nureksain data inggih punika nyorohang ring soang-soang kategorinyane. Paridabdab sane kalaksanayang sajeroning data tureksa inggih punika nyelehin data utawi reduksi data. Nyelehin data puniki kamargiang santukan data sane kapolihang pastika akeh. Usan data-data sane kapolihang punika kareduksi, kalanturang antuk ngawedarang data. Kaping untat kalanturang antuk makarya panyutet.

\section{PIKOLIH LAN TETEPASAN}

Pikolih lan tetepasan kapolihang saking bantang pikobet inggih punika wangun intrinsik miwah aji pangajahajahan tata laksana sane wenten ring pupulan satua bawak Awengi Ring Hotel Sentral kakawian I Gusti Putu Bawa Samar Gantang.

Pupulan satua bawak sane mamurda Awengi ring Hotel Sentral kakawian I Gusti Putu Samar Gantang madue 12 murda satua bawak. Sakemaon panilik wantah nilikin 5 murda satua bawak minakadi: Leak ring Bukit Pecatu, Pak Putu Joni, Kursi Roda, Oh Diani, miwah Sinengkawon. Pikolih lan tetepasan tetilik wangun intrinsik sajeroning pupulan satua bawak Awengi ring Hotel Sentral kakawian I Gusti Putu Samar Gantang katlatarang ring sor puniki.

Satua bawak kapertama sane katilikin inggih punika mamurda Leak ring Bukit Pecatu. Satua bawak puniki kapolihang wenten 7 wangun intrinsik. Unteng ring satua bawak mamurda Leak ring Bukit Pecatu puniki inggih punika madurgama, lelintihan satua bawak puniki lelintihan ka arep utawi maju. Satua bawak sane mamurda Leak ring Bukit Pecatu puniki kapolihang wenten dasa praginanyane, sakemaon wantah 7 pawatekannyane sane prasida katlatarang inggih punika pragina Titiang
(Pangawi), Made Sukada, Hillal Wahid, Suradi PW, Ngurah Parsua, Ignatius Rachmat Sukandi, miwah Maling. Pragina sane tiosan wantah kasambatang manten ring satua. Satua bawak sane mamurda Leak ring Bukit Pecatu puniki taler karereh wenten desa kala patranyane.

Desa kala patra utawi sane kabaosang latar/setting puniki kakepah dados 3 soroh inggih punika desa utawi genah, kala utawi galah, patra utawi suasana. Kawentenan desa satua bawak puniki ring Pura Tegeh Sari, ring Bukit Pecatu, ring Batan Punyan Jepune. Selanturnyane kala utawi galahnyane minakadi galah wengi, galah sanja utawi rikala sore, lan galah nuju lakar luas. Patra utawi suasananyane wenten rasa runtag krana jejeh, rasa banyol miwah sungsut.

Genah sang pengawi utawi sudut pandang ring satua bawak puniki inggih punika kagenahang ring pengawi dados pragina utama. Piteket ring satua bawak Leak ring Bukit Pecatu puniki iraga dados jatma manda tetep waspada taler majaga-jaga tekening palemahan sane kapanggihin mangda tetep polih kerahayuan. Tiosan ring punika iraga dados jatma mangda nenten enggalan jejeh. Paribasa utawi gaya bahasa ring satua bawak puniki kapolihang paribasa sasawangan, santukan wenten kruna "sakadi". Kruna "sakadi" puniki sane kaanggen cihna sasawangan, ring satua bawak puniki prasida katujuang sinalih tunggil imba utawi ketusan paribasanyane inggih punika "...pantes kaucapang ipun sakadi Bing Slamet..." (I/LBP/02/22). Prasida kacutetang yening ketusan ring ajeng puniki nyihnayang solahnyane mesib sakadi Bing Slamet.

Satua bawak kaping kalih sane katilikin mamurda Pak Putu Joni. Satua bawak sane mamurda Pak Putu Joni puniki taler kawentenang wenten 7 wangun intrinsik sane jangkep. Unteng ring satua bawak puniki kapagehan utawi kateguhan pragina drama. Lelintihan satuanyane inggih punika lelintihann ka arep utawi maju. Pragina miwah pawatekan sane katilikin wenten 3 inggih punika pragina Titiang 
(Pangawi), Ade Hendrarini, miwah Pak Putu Joni. Desa Kala Patra sane kauningin ring satua bawak Pak Putu Joni wenten genah nyane ring Genah muruk Drama, ring Pasisi Pasih Soka, lan ring Bioskop. Kala sane kauningin wenten kalih ring satua bawak puniki inggih punika galah jam 9 peteng, miwah malam minggu utawi rahina saniscara (sabtu). Selanturnyane patra sane kapolihang ring satua bawak puniki inggih punika rasa mapangenan, rasa sedih utawi sungsut, taler rasa egar utawi bagia.

Genah sang pangawi ring satua bawak mamurda Pak Putu Joni inggih punika pangawi dados pragina utama. Piteket sane kapolihang ring satua bawak puniki wenten papat inggih punika iraga dados jatma mangda madue rasa ngaat lan rasa andel rikala ngemargiang swadarman. Piteket sane kaping kalih inggih punika iraga mangda nenten sering megadang krana sering magadang nika nenten becik anggen kesehatan raga. Piteket kaping tiga iraga patut ngamolihang hiburan kapah-kapah mangda nenten ngemetuang rasa med napi malih rasa stress. Kaping untat kauningin piteketnyane inggih punika yening iraga meled gelis bisa mangda sering latihan utawi deklamasi. Paribasa utawi gaya bahasa sane kapolihang ring satua bawak puniki inggih punika sasawangan. Paribasa sasawangan sane kapolihang prasida katujuang antuk sinalih tunggil ketusan utawi imba sane wenten ring satua bawak Pak Putu Joni inggih punika "Pangawit kadepang kakupune ngigel sakadi sang manapa ngewehin widiadari sane ngoda, jantos kakupune kenyel padidi malih mararean ring samping cekceke" (II/PPJ/33/40). Dadosnyane ring ketusan ajeng nyihnahyang solah kupu-kupune sane mesib utawi mirib sakadi widiadari sane ngoda anak ngamargiang tapa.

Satua bawak sane kaping tiga katilikin mamurda Kursi Roda. Satua bawak mamurda Kursi Roda puniki taler kapolihang wangun intrinsiknyane. Unteng ring satua bawak puniki inggih punika balas budi, satua bawak puniki madue lelintihan satua ka arep utawi maju. Pragina miwah pawatekan sane kapolihang wenten 6 inggih punika pragina lbu Suarni, pragina Titiang (Pangawi), pragina Pak Putu Joni, pragina Pak Wayan Widia, pragina Ida Rufaida, miwah pragina Pak ketut Dharma Susila. Selanturnyane wenten desa kala patra sane kapolihang ring satua bawak puniki inggih punika desa utawi ganahnyane wenten tiga minakadi ring Jeron Ibu Suarni, ring Rumah Sakit lan Gedung DPR Tabanan, miwah ring Tabanan. Kala utawi galahanyane sane kaanggen ring satua bawak puniki inggih punika galah wengi. Patra utawi suasana sane wenten ring satua puniki rasa sepi kagentosin antuk rame, rasa sungsut, miwah rasa terharu utawi kalangen.

Genah sang pangawi ring satua bawak puniki pangawi dados pragina sampingan utawi pangawi dados jatma kalih ring satua. Piteket sane kapolihang ring daging satua bawak puniki inggih punika iraga dados jatma mangda stata eling tekening bantuan sane sampun kaicenin olih anak sane sampun ngewantu iraga. Paribasa sane kapolihang inggih punika sasawangan. Paribasa sasawangan puniki prasida katujuang antuk kawentenan kruna "sakadi" ring lengkaranyane. Sinalih tunggil imba utawi ketusan sane kapolihan ring satua bawak mamurda Kursi roda inggih punika "Swabawa pararaine bunder rambutnyane panjang kapusungang kuri, heling titiang ring wanin R.A. Kartini sakadi Manersti Malih" (III/KR/41/ 56). Dadosnyane ring ketusan ajeng nyihnayang lbu Suarni sane mesib utawi mirib R.A Kartini.

Satua bawak sane kaping pat mamurda Oh Diani. Satua bawak sane katilikin puniki taler kapolihan wangun intrinsik sane jangkep. Unteng ring satua bawak mamurda Oh Diani inggih punika tresna, lelintihan satuanyane inggih punika lelintihan campuhan. Pragina lan pawatekan sane kapolihang ring satua puniki wenten 7 pragina inggih punika wenten pragina Titiang (Pangawi), pragina I Gede Mangku, pragina Diani, pragina Supir Colt, pragina Bapa, pragina I Riki, miwah pragina I Batan. Selanturnyane desa kala patra sane kapolihang ring satua bawak puniki, wenten desa utawi genah inggih punika 
ring Margine Penebel-Tabanan, ring Tongose Masare, ring Tongose Malajah, miwah ring Margine tabrakan. Kala utawi galah satua bawak puniki mamargi inggih punika semengan miwah peteng. Patra ring satua bawak puniki madue rasa pengeng, patra sane sedeng kasmaran utawi seneng, rasa gedeg, galak utawi pedih, tur patra sane kapolihang malih wenten patra sedih utawi sungsut.

Genah sang pangawi sane katilikin inggih punika pangawi dados pragina utama utawi jatma utama ring satua bawak puniki. Piteket sane kawentenang ring satua bawak puniki inggih punika iraga dados jatma mangda nenten mageson-gesonan ring margine yening madue rasa sungsut, pedih, tur sebet mangda nenten ngemetuang sengkala. Paribasa sane kapolihang wantah sasawangan. Sinalih tunggil ketusan utawi imba sasawangan sane kapolihang ring satua bawak puniki inggih punika "Tampis wengi makire pules, Gede Mangku satata ngarimikang ane madan Diani, taruni magingsir saking Yogya punika. Samalinne makisikisi nyambat adan Diani punika, matane kidemange sarwa limane masidakep. Tulya kadi anake mayoga. Wantah sapunika indiknyane" (IV/OD/47/80). Lengkara sane kagaris bawahin punika katujuang paribasa sasawangan, sane madue artos nyihnayang Gede Mangku sane mesib cara anake sane nglaksanayang samadi.

Satua bawak sane kaping untat sane katilikin mamurda Sinengkawonan. Wangun intrinsik sane kapolihang wenten papitu. Minakadi unteng ring satua bawak puniki inggih punika semangat sang guru, lelintihan satua bawak puniki inggih punika ka arep. Pragina lan pawatekan sane kapolihan ring satua bawak puniki wenten 6 pragina inggih punika pragina Putu Munu, pragina Luh Widarni, pianakpianaknyane, guru-gurunyane, muridmuridnyane, miwah dokternyane.

Desa Kala Patra sane kauningin ring satua bawak sinengkawonan puniki, minakadi desa utawi genahnyane inggih punika ring Kori Umah, ring Margane, ring Apotik Sehat ring Sanglah, lan ring
Sekolah. Kala utawi galahnyane limang tahun, lan semengan. Patra utawi suasana sane kapolihang inggih punika rasa bangga utawi rasa bagia, miwah rasa sungkan. Genah sang pangawi sane katilikin inggih punika pangawi dados jatma kaping tiga ring satua. Piteket-piteket sane kapolihang ring satua bawak mamurda Sinengkawonan puniki inggih punika iraga pinaka jatma patut semangat makarya, stata eling mabakti kapining Ida Sang Hyang Widhi Wasa, lan nyenengin pakaryan sane kakaryanin. Selanturnyane paribasa sane kapolihang inggih punika sasawangan. Paribasa sasawangan puniki prasida katujuang antuk ketusan utawi imbanyane inggih punika "manah Putu Munu sakadi kasiratin toya sawindu nampi kenyem kurenane," (V/SK/74/97). Ring ketusan puniki wenten kruna "sakadi" sane nyihnayang cihna ring sasawangan. Ketusan ring ajeng puniki madue artos manah Putu Munu sane liang pisan santukan manyingakin kenyem kurenane sane manis.

Aji pangajah-ajahan tata laksana puniki prasida kapolihang ring pupulan satua bawak sane mamurda Awengi ring Hotel Sentral kekawian I Gusti Bawa Samar Gantang. Murda satua bawak sane wenten inggih punika 12 murda satua bawak, sakemaon sane katilikin aji pangajah-ajahan tata laksananyane wantah 5 murda satua bawak inggih punika Leak ring Bukit Pecatu, Pak Putu Joni, Kursi Roda, Oh Diani, miwah Sinengkawonan.

Satua bawak sane katilikin kapertama antuk aji pangajah-ajahan tata laksananyane inggih punika Leak ring Bukit Pecatu kapolihang wenten 5 minakadi sradha bhakti, seneng ngwacen, pasawitran, arjawa, miwah meled uning. Satua bawak kaping kalih mamurda Pak Putu Joni kapolihang aji pangajah-ajahan tata laksana wenten 5 inggih punika meled uning, nganutin swadarman, jemet makarya, masawitra, miwah awiwahara. Satua bawak sane kaping tiga mamurda Kursi Roda kapolihang wenten 2 aji pangajah-ajahan tata laksana inggih punika ngajiang kawagedan miwah seneng ngewacen.Satua kaping pat mamurda 
Oh Diani kapolihang wenten 1 aji pangajah-ajahan tata laksana inggih punika masawitra. Satua kaping untat mamurda sinengkawonan kapolihang taler 4 aji pangajah-ajahan tata laksana inggih punika sradha bhakti, jemet makarya, arjawa, miwah tresna asih. Makasami aji pangajah-ajahan tata laksana sane kawentenang ring 5 murda satua bawak ring ajeng sampun katujuang antuk ketusan satuanyane.

\section{PAMUPUT}

Malarapan antuk pikolih lan tetepasan sane sampun kawedarang ring ajeng prasida kacutetang inggih punika satua bawak sane katilikin wantah 5 murda satua bawak minakadi Leak ring Bukit Pecatu, Pak Putu Joni, Kursi Roda, Oh Diani, miwah Sinengkawonan. Satua bawak sane katilikin puniki madue 7 wangun intrinsik sane jangkep. Wangun Intrinsiknyane minakadi unteng, lelintihan, pragina miwah pawatekan, desa kala patra, genah sang pangawi, piteket lan paribasa.

5 murda satua bawak sane katilikin puniki taler madue aji pangajahajahan tata laksana. Aji pangajah-ajahan tata laksana sane kapolihang inggih punika sradha bhakti, seneng ngawacen, masawitra, arjawa, meled uning, nganutin swadarman, jemet makarya, awiwahara, ngajiang kawagedan, miwah tresna.

Piteket ring sajeroning tatilikan puniki inggih punika pangaptin panilik majeng pangwacen, mangda stata seneng ngwacen pupulan satua bawak antuk karesepang, kaplajahin taler kalaksanayang aji pangajah-ajahan tata laksana sane patut. Piteket kaping kalih katurang majeng krama Bali mangda sareng nglastariang kasusastraan Bali anyar sinalih tunggilnyane satua bawak. Santukan satua bawak prasida kakaryanin lan prasida kasatuang olih parajana Bali punika. Yening nenten ngawit saking parajana Bali satua bawak sane madaging aji pangajah-ajahan tata laksana puniki nenten prasida ngelimbak tur kria sastra puniki sayan-sayan ngareredang. Piteket selanturnyane majeng panilik sane lianan, tetilikan sane kalaksanayang puniki sinah doh saking paripurna, santukan akeh wenten kakirangannyane. Tetilikan puniki wantah nilikin indik kawentenan wangun intrinsik miwah aji pangajah-ajahan tata laksana sane wenten ring daging buku pupulan satua bawak Awengi ring Hotel Sentral. Panilik ngaptiang majeng ring panilik lianan mangda ngalimbakang malih indik daging tetilikan sane mapaiketan sareng wangun intrinsik miwah aji pangajah-ajahan tata laksana ring pupulan satua bawak sane tiosan.

\section{KAPUSTAKAAN}

Antara, I Gusti Putu.2009. Frosa Fiksi Bali Tradisional. Balai Bahasa Denpasar.

2011. Frosa Fiksi Bali. Singaraja. Yayasan Gita Wandawa.

Gantang, I Gusti Bawa Samar. 2009. Pupulan Satua Cutet Basa Bali Anyar Awengi ring Hotel Sentral. Surabaya: Paramita.

Gautama, Wayan Budha. 2007. Kesusastraan Bali. Cakepan Penuntun Mlajahin Kasusastraan Bali. Gianyar: Paramitha Surabaya.

--------. 2007. Kamus Bahasa Bali. Kamus Bali-Indonesia. Paramitha Surabaya

Nurgiantoro, Burhan. 1998. Teori Pengkajian Fiksi. Yogyakarta: Gadjah Mada University Press

Sumardjo, J. \& Saini K. M. 1997. Apresiasi Kesusasteraan. Jakarta: PT Gramedia Pustaka Utama.

Sumardjo, Jacob. 2001. Beberapa Petunjuk Menulis Cerpen. Bandung: Mitra.

Suroto. 1989. Apresiasi Sastra Indonesia. Jakarta: Erlangga.

Sutjaja, Gusti Made. 2006. Kamus BaliIndonesia. Denpasar: Lotus Widya Suari

Wendra, I Wayan. 2013. Buku Ajar Penulisan Karya IImiah. Singaraja: Undiksha. 
Yaumi, Muhammad.2014. Pendidikan

Karakter. Jakarta: Prenadamedia

Group. 